Review

\title{
New Developments of Gastric Cancer Biomarker Research
}

\author{
Hualin $\mathrm{Fu}^{1,2}$ \\ ${ }^{1}$ Institute of Nano Biomedicine and Engineering, Key Laboratory for Thin Film and Microfabrication of the Ministry of Education, \\ Department of Instrument Science and Engineering, School of Electronic Information and Electrical Engineering, Shanghai Jiao Tong \\ University, 800 Dongchuan Road, Shanghai 200240, China. \\ ${ }^{2}$ National Center for Translational Medicine, Shanghai Jiao Tong University, 800 Dongchuan Road, Shanghai 200240, China..
}

Corresponding author. E-mail: hfu@sjtu.edu.cn; Tel: +86-21-34206375

Received: Sep. 27, 2016; Accepted: Nov. 18, 2016; Published: Dec. 6, 2016.

Citation: Hualin Fu, New Developments of Gastric Cancer Biomarker Research. Nano Biomed. Eng., 2016, 8(4): 268-273.

DOI: $10.5101 /$ nbe.v8i4.p268-273.

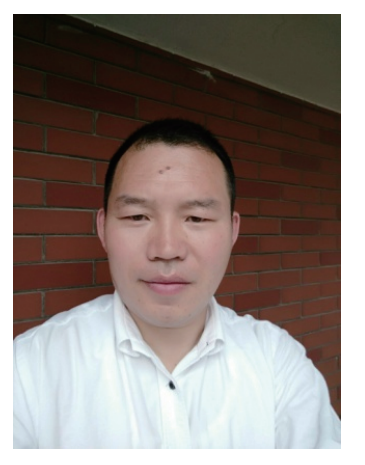

Dr. Hualin Fu obtained his PhD from the University of Southern California in 2007. He did his post-doctoral fellow training in UCLA Hillbolom Islet Research Center from 2007 to 2009. He is now an Assistant Professor at Shanghai Jiao Tong University. His current research interests include epigenetic regulation specifically TET1 and p53-EZH2 pathway in gastric carcinogenesis, the relation between H. Pylori and EBV infections and gastric cancer and the effect of immune cell microenvironment on gastric cancer.

\begin{abstract}
Gastric cancer is a deadly disease with high incidence and mortality rate in China. Early detection and treatment of gastric cancer showed significantly better 5-year survival rate. Conventional screening methods of gastric cancer include barium meal or endoscopic screening. There is a great need to find new biomarkers of gastric cancer for simpler, faster, and non-invasive screening of gastric cancer. A large array of molecules such as protein markers, metabolite markers, RNA markers, breath organic molecules have been identified as potential markers of gastric screening. Among them, pepsinogens and gastrin-17 have been applied in large scale of population screening. New species of metabolite markers, microRNA markers and breath molecules may further enhance the simplicity, sensitivity and specificity of gastric cancer screening.
\end{abstract}

Keywords: Gastric cancer; Biomarker; Protein; RNA; Volatile organic compound; Metabolite

\section{Introduction}

Gastric cancer is the No. 2 cancer in China by incidence [1]. The survival rate of gastric cancer patients is often low because of the late detection of the disease [2]. Methods are urgently needed to screen patients with possible gastric cancer diseases at early stage of development. The purpose of gastric cancer 
biomarker research is to find early markers of gastric cancer with possible applications of gastric cancer early detection. Traditional gastric cancer biomarkers are usually serum protein markers. Today, the definition of cancer biomarkers is extended to other type of molecules such as RNAs, small molecular metabolites or even volatile organic gas molecules from breath (Fig. 1). In this short review, we will summarize the recent progress on the gastric cancer biomarker discovery.

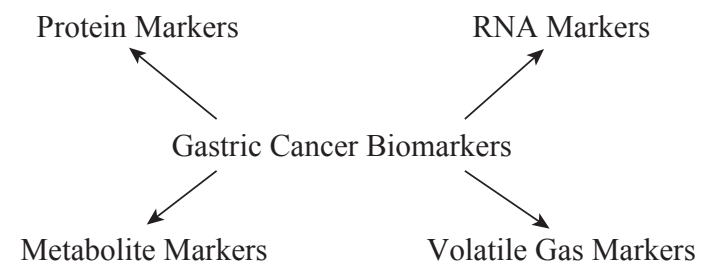

Fig. 1 Summary of gastric cancer biomarkers.

\section{Serum Protein Biomarkers of Gastric Cancer}

Serum protein biomarkers of gastric cancer can be divided by gastric cancer-specific markers and general tumor markers. A group of serum protein biomarkers of gastric cancer are gastric tissue specific or related to gastric-specific infections. Proteins such as Pepsinogen I (PGI, also called PGA), Pepsinogen II (PGII, also called PGC) and Gastrin 17 are considered specific markers of gastric cancer because of their gastric specific gene expression. Pepsinogens are the precursor of pepsins, the main proteases produced by the stomach. Since PGs are rarely expressed in other organs, so pepsinogen level can reflect the function of the stomach. The decrease PGI level is an important indicator of gastric cancer progression. In clinical practice, PGI measurement often needs to be used in combination with other indicators. The final diagnostic results need to be confirmed with $\mathrm{X}$ ray or endoscopy to verify whether the patients are suffering from gastric cancer [3, 4]. Gastrin 17 is a truncated peptide of the gastrin protein, a hormone secreted by the gastric antrum, which is important for gastric acid secretion[5]. Pepsinogens mainly produced from the the upper part of gastric body while gastrin 17 mainly secreted by the bottom half of the gastric body. Measurement of Pepsinogens and Gastrin17 can detect the specific positions of gastritis [6]. TFF3 (Trefoil factor 3 ) is a protein mainly expressed in goblet cells of intestine and colon. However, TFF3 positive goblet-like cells are found in intestinal metaplasia, an important precancerous stage of gastric cancer. For this reason, TFF3 level quantitation might help with gastric cancer detection [7, 8]. H. Pylori antibodies, CagA antibodies, anti-parietal cell antibodies are related to gastric specific infections, which reflect ongoing or past gastric infections that could be related to gastric cancer development [7, 9-13]. These gastric disease related antibodies are useful biomarkers for assessing gastric cancer risk.

Literature search also found many other proteins considered as gastric cancer screening markers although most of them are not specific to gastric cancer. These proteins are either general tumor markers or proteins that are associated with signaling related to carcinogenesis such as cell proliferation, angiogenesis or inflammation. These proteins include CEA (Carcinoembryonic Antigen) ([14], CA125 (Cancer Antigen 125) [14], CA19-9 (Cancer Antigen 19-9) [14, 15], AFP (Alpha-Fetoprotein) [16, 17], Pyruvate M2 Kinase [15, 18], serum amyloid A $[19,20]$, macrophage migration inhibitory factor [21, 22], Leptin [23], dickkopf [24, 25], olfactomedin 4 [26], VAP-1 [27, 28], UPA [29, 30], Cathepsin B [30-32], HMW kininogen [33], P53 antibody [34, 35], cytokeratin 18 [36-38], RegIV [39], IPO-38 [40], S100A6 [41], thrombin light chain [42], fibrinopeptide A [43, 44], angiopoietin-like protein 2 [45-47]. CEA, CA125, CA19-9, AFP, M2 Pyruvate Kinase are common tumor markers also overexpressing in other type of cancers [48-54]. p53 antibodies usually indicates $\mathrm{p} 53$ gene mutations, which also happened in many type of cancers [55]. cytokeratin 18, RegIV are apoptosis related proteins indicating cell death during gastric cancer development [56, 57]. Other proteins such as serum amyloid A, macrophage migration inhibitory factor, Leptin, dickkopf, olfactomedin 4, VAP-1, UPA, Cathepsin B, HMW kininogen, IPO-38, S100A6, angiopoietin-like protein 2, thrombin light chain and fibrinopeptide A might be related to cancerrelated inflammation, extracellular matrix remodeling, angiogenesis and blood coagulation abnormalities.

Protein biomarkers of gastric cancer have been applied in large scale population screening in Japan and Korea. In A Japanese study, pepsinogen screening, with PGI $<70 \mathrm{ng} / \mathrm{ml}$, and PGI:PGII $<3.0$ as the cutoff value, could detect gastric cancer with the sensitivity of $84.6 \%$ and specificity of $73.6 \%$ [58]. A South Korea study using PGI:PGII $<3.0$ as an indicator for gastric cancer, a detection sensitivity of $59.2 \%$ and specificity of $61 \%$ was achieved [3]. Although the pepsinogen serum screening appeared to be effective in gastric 
cancer screening, the specificity and sensitivity of detection still needs to be improved. There is great difference among the Japanese or Korean studies using the same pepsinogen assay, which indicates that there are possible confounding factors, such as ethnicities or diet and social habits, which may affect the outcome of pepsinogen screening. It is necessary to develop some new methods for serum screening of gastric cancer.

\section{Metabolic Markers of Gastric Cancer}

The most notable metabolic difference between cancer cells and normal cells is that cancer cells relies heavily on glycolysis for energy while normal cells rely on oxidative phosphorylation, a phenomenon called Warbug effect [59]. The level of lactate, a byproduct of glucose glycolysis, was constantly found to be increased in gastric patient serum or tissue samples $[60,61]$. Cancer cells also have a high rate of protein synthesis. Accordingly, many metabolic studies showed an increase of amino acids, such as glycine, methionine, asparagine, aspartate and tyrosine in gastric cancer patients [60]. Cancer cells also have a high rate of nucleotide synthesis for the increasing demands of DNA synthesis and DNA repair. Reports also suggested altered nucleotide metabolites in certain type of cancers [62-64]. Few researches studied the fatty acid metabolism metabolites in gastric cancer patients. However, both increased fatty acid synthesis (FASN) and increased fatty acid oxidation (CPT1A) have been associated with cancer development [65-67]. Fatty acid oxidation metabolites such as acetone and $\beta$-hydroxybutyrate have been identified as potential biomarkers of gastric cancer [68, 69].

\section{RNA Markers of Gastric Cancer}

Traditionally, RNA is considered an unstable species of biomolecules, thus unsuitable to be used as biomarkers for cancer. However, recent research suggested some serum non-coding RNA could also be potential gastric specific markers. Long non-coding RNA HULC and H19 were reported to be promising novel biomarkers in plasma of patients with gastric cancer [70-75]. MicroRNA is a relatively stable species of RNA in the serum. Potential serum microRNA biomarkers included miR-187, miR-371-5p and miR378, microRNA-21, miR-195, miRNA-206, MiR- 16-5p and MiR-19b-3p. Serum miR-187, miR-371$5 p$ and miR-378, microRNA-21, miR-627, miR-629 and miR-652 levels are positively related to gastric cancer while serum miR-195, miRNA-206, MiR-16$5 p$ and MiR-19b-3p levels are negatively correlated with gastric cancer [76-81]. miR-378 alone could discriminate GC patients from healthy controls with $87.5 \%$ sensitivity and $70.73 \%$ specificity. High levels of miR-21 were associated with an increased tumor size and an advanced $\mathrm{pT}$ stage. Low expression of miR-195 is a potential marker for gastric cancer. Serum miRNA-206 was down-regulated in GC patients compared with healthy controls. An important issue in using microRNA as a tumor biomarker is to choose a correct reference gene. Analysis showed that miR-16 and miR-93 were the most stably expressed reference genes for serum microRNA analysis of gastric cancer patients and healthy controls [82].

\section{Volatile Organic Compounds as Markers of Gastric Cancer}

Breath analysis is an another appealing method for cancer detection because of its convenient, painless, noninvasive way of sampling. Several studies have highlighted the potential of breath analysis for gastric cancer detection. The identified volatile organic compounds from gastric cancer patients included hexanoic acid, phenol, methyl phenol, ethyl phenol, 2-propenenitrile, 2-butoxy-ethanol, furfural, 6-methyl5-hepten-2-one, hexadecane, 4-methyloctane, 1,2,3-trimethylbenzene, $\alpha$-methyl-styrene, acetone, tetradecane, 3-methylpentane, hexane, 2,3-dimethylpentane, 2-methylhexane, and dodecane [83-86]. In addition, 2-butanone and menthol have been found at lower levels in gastric cancer patients comparing to controls. Using a combination of 4 VOCs, gastric cancer patients could be differentiated from gastritis patients (the integrated area under the ROC curve is 0.91) [69]. While with a model using a combination of 8 VOCs, gastric cancer patients could be discriminated from precancerous gastritis controls with $73 \%$ sensitivity, $98 \%$ specificity and $92 \%$ accuracy [84]. It has been a concern that breath analysis might be confounded by H. pylori infection or smoking behaviors. However, recent study showed that breath analysis with a breathsensing nanoarray was not affected by ages, sex, smoking, drinking habits, H. pylori infection or proton pump inhibitor medications [84]. 


\section{Conclusions}

New biomarkers have been continuously discovered for gastric cancer early detection. However, protein biomarkers of gastric cancer are the only kind of biomarkers that go into clinical applications. Protein markers are relatively easy to detect and proteins such as pepsinogens are closely related to gastric physiology, which makes them easier to adopt in clinic applications. Metabolic biomarkers are also easy to detect in the patient blood, however, their specificities to gastric cancer has not been firmly established. RNA biomarkers may help to elucidate more detailed mechanisms of gastric cancer while their analysis is more complicate than the analysis of protein biomarkers. MicroRNAs might be useful biomarkers for gastric cancer detection because of their stability in the patient plasma. Breath biomarkers have the advantage of easy sampling but the analysis of breath biomarkers might be more expensive than other biomarkers. Nonetheless, metabolic biomarkers, RNA biomarkers and breath biomarkers hold great promise for the future of gastric cancer early detection.

\section{Acknowledgements}

This work was supported by National Natural Science Foundation of China No. 81472235.

\section{References}

[1] R. Zheng, S. Zhang, and L. Wu, Report of Incidence and Mortality from China Cancer Registries in 2008. China Cancer, 2012, 21(1): 1-12.

[2] N. Howlader, A.M. Noone, M. Krapcho, et al. (eds), SEER cancer statistics review, 1975-2011. National Cancer Institute. Bethesda, 2014.

[3] J.M. Kang, N. Kim, J.Y. Yoo, et al., The role of serum pepsinogen and gastrin test for the detection of gastric cancer in Korea. Helicobacter, 2008, 13(2): 146-156.

[4] N. Kim, H.C. Jung, The role of serum pepsinogen in the detection of gastric cancer. Gut Liver, 2010, 4(3): 307319.

[5] J.S. Edkins, The chemical mechanism of gastric secretion. J Physiol, 1906, 34(1-2): 133-144.

[6] R. Kikuchi, Y. Abe, K. Iijima, et al., et al., Low serum levels of pepsinogen and gastrin 17 are predictive of extensive gastric atrophy with high-risk of early gastric cancer. Tohoku J Exp Med, 2011, 223(1): 35-44.

[7] M. Kaise, J. Miwa, A. Fujimoto, et al., Influence of Helicobacter pylori status and eradication on the serum levels of trefoil factors and pepsinogen test: serum trefoil factor 3 is a stable biomarker. Gastric Cancer, 2013, 16(3): 329-337.

[8] M. Kaise, J. Miwa, J. Tashiro, et al., The combination of serum trefoil factor 3 and pepsinogen testing is a valid non-endoscopic biomarker for predicting the presence of gastric cancer: a new marker for gastric cancer risk. $J$ Gastroenterol, 2011, 46(6): 736-745.

[9] S. Kikuchi, Y. Abe, K. Iijima, K. et al., Association between infections with CagA-positive or -negative strains of Helicobacter pylori and risk for gastric cancer in young adults. Research Group on Prevention of Gastric Carcinoma Among Young Adults. Am J Gastroenterol, 1999, 94(12): 3455-3459.

[10] K. Miki, Gastric cancer screening by combined assay for serum anti-Helicobacter pylori IgG antibody and serum pepsinogen levels - "ABC method". Proc Jpn Acad Ser B Phys Biol Sci, 2011, 87(7): 405-414.

[11] S. Shiota, O. Matsunari, M. Watada, et al., Serum Helicobacter pylori CagA antibody as a biomarker for gastric cancer in east-Asian countries. Future Microbiol, 2010, 5(12): 1885-1893.

[12] J. Parsonnet, G.D. Friedman, N. Orentreich, et al., Risk for gastric cancer in people with CagA positive or CagA negative Helicobacter pylori infection. Gut, 1997, 40(3): 297-301.

[13] K. Sugiu, T. Kamada, M. Ito, et al., Anti-parietal cell antibody and serum pepsinogen assessment in screening for gastric carcinoma. Dig Liver Dis, 2006, 38(5): 303307.

[14] G.I. Hwang, H.Y. Chang, H.S. Byung, et al., Predictive value of preoperative serum CEA, CA19-9 and CA125 levels for peritoneal metastasis in patients with gastric carcinoma. Cancer Res Treat, 2004, 36(3): 178-181.

[15] J. Schneider, G. Schulze, Comparison of tumor M2pyruvate kinase (tumor M2-PK), carcinoembryonic antigen (CEA), carbohydrate antigens CA 19-9 and CA 72-4 in the diagnosis of gastrointestinal cancer. Anticancer Res, 2003, 23(6D): 5089-5093.

[16] H.J. Lin, Y.H. Hsieh, W.L. Fang, et al., Clinical manifestations in patients with alpha-fetoproteinproducing gastric cancer. Curr Oncol, 2014, 21(3): e394-e399.

[17] X. Liu, W. Sheng, and Y. Wang, An analysis of clinicopathological features and prognosis by comparing hepatoid adenocarcinoma of the stomach with AFPproducing gastric cancer. J Surg Oncol, 2012, 106(3): 299-303.

[18] Y. Kumar, N. Tapuria, N. Kirmani, et al., Tumour M2pyruvate kinase: a gastrointestinal cancer marker. Eur $J$ Gastroenterol Hepatol, 2007, 19(3): 265-276.

[19] D.C. Chan, C.J. Chen, H.C. Chu, et al., Evaluation of serum amyloid A as a biomarker for gastric cancer. Ann Surg Oncol, 2007, 14(1): 84-93.

[20] S.A. Moshkovskii, Why do cancer cells produce serum amyloid A acute-phase protein? Biochemistry (Mosc), 2012, 77(4): 339-341.

[21] F. Tas, S. Karabulut, M. Serilmez, et al., Serum levels of macrophage migration-inhibitory factor (MIF) have diagnostic, predictive and prognostic roles in epithelial ovarian cancer patients. Tumour Biol, 2014, 35(4): 33273331.

[22] H.H. Xia, Y. Yang, K.M. Chu, et al., Serum macrophage migration-inhibitory factor as a diagnostic and prognostic biomarker for gastric cancer. Cancer, 2009, 115(23): 5441-5449.

[23] L.G. Capelle, A.C. de Vries, J. Haringsma, et al., Serum levels of leptin as marker for patients at high risk of gastric cancer. Helicobacter, 2009, 14(6): 596-604.

[24] C. Gao, R. Xie, C. Ren, et al., Dickkopf-1 expression is a novel prognostic marker for gastric cancer. J Biomed Biotechnol, 2012, 2012: 804592.

[25] H.S. Lee, H.E. Lee, D.J. Park, et al., Clinical significance of serum and tissue Dickkopf-1 levels in patients with gastric cancer. Clin Chim Acta, 2012, 413(21-22): 1753- 
1760.

[26] L. Yu, L. Wang, and S. Chen, Olfactomedin 4, a novel marker for the differentiation and progression of gastrointestinal cancers. Neoplasma, 2011, 58(1): 9-13.

[27] M.A. Kaplan, M. Kucukoner, A. Inal, et al., Relationship between serum soluble vascular adhesion protein-1 level and gastric cancer prognosis. Oncol Res Treat, 2014, 37(6): 340-344.

[28] H. Yasuda, Y. Toiyama, M. Ohi, et al., Serum soluble vascular adhesion protein-1 is a valuable prognostic marker in gastric cancer. J Surg Oncol, 2011, 103(7): 695699.

[29] N. Harbeck, M. Schmitt, M. Vetter, et al., Prospective Biomarker Trials Chemo N0 and NNBC-3 Europe Validate the Clinical Utility of Invasion Markers uPA and PAI-1 in Node-Negative Breast Cancer. Breast Care (Basel), 2008. 3(s2): 11-15.

[30] L. Herszenyi, G. Istvan, R. Cardin, et al., Serum cathepsin $\mathrm{B}$ and plasma urokinase-type plasminogen activator levels in gastrointestinal tract cancers. European Journal of Cancer Prevention, 2008, 17(5): 438-445.

[31] M.P. Ebert, S. Kruger, M.L. Fogeron, et al., Overexpression of cathepsin B in gastric cancer identified by proteome analysis. Proteomics, 2005, 5(6): 1693-1704.

[32] T. Hirano, H. Yoshioka, Serum cathepsin B levels, urinary excretion of cathepsin B and tissue cathepsin B content in the patients with gastric cancer. Nihon Geka Hokan, 1993, 62(5): 217-221.

[33] H. Umemura, A. Togawa, K. Sogawa, et al., Identification of a high molecular weight kininogen fragment as a marker for early gastric cancer by serum proteome analysis. J Gastroenterol, 2011. 46(5): 577-585.

[34] K. Shimizu, Y. Ueda, and H. Yamagishi, Titration of serum p53 antibodies in patients with gastric cancer: a single-institute study of 40 patients. Gastric Cancer, 2005, 8(4): 214-219.

[35] A. Suppiah, J. Greenman, Clinical utility of anti-p53 auto-antibody: systematic review and focus on colorectal cancer. World J Gastroenterol, 2013, 19(29): 4651-4670.

[36] T. Abe, M. Fukumoto, K. Tsuchiya, et al., Human monoclonal antibodies against cytokeratin 18 generated from patients with gastric cancer. Jpn J Cancer Res, 1989, 80(3): 271-276.

[37] C. Caulin, G.S. Salvesen, and R.G. Oshima, Caspase cleavage of keratin 18 and reorganization of intermediate filaments during epithelial cell apoptosis. J Cell Biol, 1997, 138(6): 1379-1394.

[38] K. Oyama, S. Fushida, J. Kinoshita, et al., Serum cytokeratin 18 as a biomarker for gastric cancer. Clin Exp Med, 2013. 13(4): 289-295.

[39] Y. Mitani, N. Oue, S. Matsumura, et al., Reg IV is a serum biomarker for gastric cancer patients and predicts response to 5-fluorouracil-based chemotherapy. Oncogene, 2007, 26(30): 4383-4393.

[40] Y. Hao, Y. Yu, L. Wang, et al., IPO-38 is identified as a novel serum biomarker of gastric cancer based on clinical proteomics technology. J Proteome Res, 2008, 7(9): 36683677.

[41] J. Zhang, K. Zhang, X. Jiang, et al., S100A6 as a potential serum prognostic biomarker and therapeutic target in gastric cancer. Dig Dis Sci, 2014, 59(9): 2136-2144.

[42] M.P. Ebert, S. Lamer, J. Meuer, et al., Identification of the thrombin light chain a as the single best mass for differentiation of gastric cancer patients from individuals with dyspepsia by proteome analysis. J Proteome Res, 2005, 4(2): 586-590.

[43] M.P. Ebert, D. Niemeyer, S.O. Deininger, et al., Identification and confirmation of increased fibrinopeptide a serum protein levels in gastric cancer sera by magnet bead assisted MALDI-TOF mass spectrometry. $J$
Proteome Res, 2006, 5(9): 2152-2158.

[44] A. Sagripanti, A. Carpi, M. Ferdeghini, et al., The measurement of plasma fibrinopeptide A in breast cancer patients. Rays, 1987, 12(2): 65-69, 107-109.

[45] T. Yoshinaga, T. Shigemitsu, H. Nishimata, et al., Angiopoietin-like protein 2 is a potential biomarker for gastric cancer. Mol Med Rep, 2015, 11(4): 2653-2658.

[46] Y. Toiyama, K. Tanaka, T. Kitajima, et al., Serum angiopoietin-like protein 2 as a potential biomarker for diagnosis, early recurrence and prognosis in gastric cancer patients. Carcinogenesis, 2015. 36(12): 1474-1483.

[47] T. Shimura, Y. Toiyama, K. Tanaka, et al., Angiopoietinlike Protein 2 as a Predictor of Early Recurrence in Patients After Curative Surgery for Gastric Cancer. Anticancer Res, 2015, 35(9): 4633-4639.

[48] J. Jarvisalo, M. Hakama, P. Knekt, et al., Serum tumor markers CEA, CA 50, TATI, and NSE in lung cancer screening. Cancer, 1993, 71(6): 1982-1988.

[49] B.L. Strom, G. Maislin, S.L. West, et al., Serum CEA and CA 19-9: potential future diagnostic or screening tests for gallbladder cancer? Int J Cancer, 1990, 45(5): 821-824.

[50] S. Alberico, M.C. Facca, R. Millo, et al., Tumoral markers (CA 125-CEA) in the screening of ovarian cancer. Eur $J$ Gynaecol Oncol, 1988. 9(6): 485-489.

[51] Y.C. Kim, J.H. Kim, D.Y. Cheung, et al., The Usefulness of a Novel Screening Kit for Colorectal Cancer Using the Immunochromatographic Fecal Tumor M2 Pyruvate Kinase Test. Gut Liver, 2015, 9(5): 641-648.

[52] C. Tonus, M. Sellinger, K. Koss, et al., Faecal pyruvate kinase isoenzyme type M2 for colorectal cancer screening: a meta-analysis. World J Gastroenterol, 2012, 18(30): 4004-4011.

[53] M. Abdullah, A.A. Rani, M. Simadibrata, et al., The value of fecal tumor M2 pyruvate kinase as a diagnostic tool for colorectal cancer screening. Acta Med Indones, 2012, 44(2): 94-99.

[54] W. Meng, H.H. Zhu, Z.F. Xu, et al., Serum M2-pyruvate kinase: A promising non-invasive biomarker for colorectal cancer mass screening. World J Gastrointest Oncol, 2012, 4(6): 145-151.

[55] M. Iwamuro, Y. Kawai, T. Matsumoto, et al., Serum anti-p53 antibody as a tumour marker for colorectal cancer screening. Ecancermedicalscience, 2015, 9: 560.

[56] C. Ausch, V. Buxhofer-Ausch, U. Olszewski, et al., Caspase-cleaved cytokeratin 18 fragment (M30) as marker of postoperative residual tumor load in colon cancer patients. Eur J Surg Oncol, 2009, 35(11): 11641168.

[57] M. Ramazan Sekeroglu, S. Aydin, H. Dulger, et al., Diagnostic value of cytokeratin-18 as a tumor marker in bladder cancer. Clin Biochem, 2002, 35(4): 327-331.

[58] F. Kitahara, K. Kobayashi, T. Sato, et al., Accuracy of screening for gastric cancer using serum pepsinogen concentrations. Gut, 1999, 44(5): 693-697.

[59] M.V. Liberti, J.W. Locasale, The Warburg Effect: How Does it Benefit Cancer Cells? Trends Biochem Sci, 2016, 41(3): 211-218.

[60] A. Hirayama, K. Kami, M. Sugimoto, et al., Quantitative metabolome profiling of colon and stomach cancer microenvironment by capillary electrophoresis time-offlight mass spectrometry. Cancer Res, 2009, 69(11): 49184925.

[61] N. Abbassi-Ghadi, S. Kumar, J. Huang, et al., Metabolomic profiling of oesophago-gastric cancer: a systematic review. Eur J Cancer, 2013, 49(17): 36253637.

[62] M. Meuth, The molecular basis of mutations induced by deoxyribonucleoside triphosphate pool imbalances in mammalian cells. Exp Cell Res, 1989, 181(2): 305-316.

[63] L. Chang, R. Guo, Q. Huang, et al., Chromosomal 
instability triggered by Rrm $2 b$ loss leads to IL-6 secretion and plasmacytic neoplasms. Cell Reports, 2013, 3(5): 1389-1397.

[64] G. Weinberg, B. Ullman, and D.W. Martin, Jr., Mutator phenotypes in mammalian cell mutants with distinct biochemical defects and abnormal deoxyribonucleoside triphosphate pools. Proceedings of the National Academy of Sciences of the United States of America, 1981, 78(4): 2447-2451.

[65] E. Rysman, K. Brusselmans, K. Scheys, et al., De novo lipogenesis protects cancer cells from free radicals and chemotherapeutics by promoting membrane lipid saturation. Cancer Res, 2010, 70(20): 8117-8126.

[66] F.P. Kuhajda, K. Jenner, F.D. Wood, et al., Fatty acid synthesis: a potential selective target for antineoplastic therapy. Proceedings of the National Academy of Sciences of the United States of America, 1994, 91(14): 6379-6383.

[67] Q. Qu, F. Zeng, X. Liu, et al., Fatty acid oxidation and carnitine palmitoyltransferase I: emerging therapeutic targets in cancer. Cell Death Dis, 2016, 7: e2226.

[68] L. Yu, J. Aa, J. Xu, et al., Metabolomic phenotype of gastric cancer and precancerous stages based on gas chromatography time-of-flight mass spectrometry. $J$ Gastroenterol Hepatol, 2011, 26(8): 1290-1297.

[69] S. Kumar, J. Huang, J.R. Cushnir, et al., Selected ion flow tube-MS analysis of headspace vapor from gastric content for the diagnosis of gastro-esophageal cancer. Anal Chem, 2012, 84(21): 9550-9557.

[70] C. Jin, W. Shi, F. Wang, et al., Long non-coding RNA HULC as a novel serum biomarker for diagnosis and prognosis prediction of gastric cancer. Oncotarget, 2016.

[71] Y. Zhao, Q. Guo, J. Chen, et al., Role of long noncoding RNA HULC in cell proliferation, apoptosis and tumor metastasis of gastric cancer: a clinical and in vitro investigation. Oncol Rep, 2014, 31(1): 358-364.

[72] J.S. Chen, Y.F. Wang, X.Q. Zhang, et al., H19 serves as a diagnostic biomarker and up-regulation of $\mathrm{H} 19$ expression contributes to poor prognosis in patients with gastric cancer. Neoplasma, 2016, 63(2): 223-230.

[73] X. Zhou, C. Yin, Y. Dang, et al., Identification of the long non-coding RNA H19 in plasma as a novel biomarker for diagnosis of gastric cancer. Sci Rep, 2015, 5: 11516.

[74] H. Li, B. Yu, J. Li, et al., Overexpression of lncRNA H19 enhances carcinogenesis and metastasis of gastric cancer. Oncotarget, 2014, 5(8): 2318-2329.

[75] E.B. Zhang, L. Han, D.D. Yin, et al., c-Myc-induced, long, noncoding $\mathrm{H} 19$ affects cell proliferation and predicts a poor prognosis in patients with gastric cancer. Med Oncol, 2014, 31(5): 914.
[76] H. Liu, L. Zhu, B. Liu, et al., Genome-wide microRNA profiles identify miR-378 as a serum biomarker for early detection of gastric cancer. Cancer Lett, 2012, 316(2): 196-203.

[77] J. Song, Z. Bai, J. Zhang, et al., Serum microRNA-21 levels are related to tumor size in gastric cancer patients but cannot predict prognosis. Oncol Lett, 2013, 6(6): 1733-1737.

[78] Y.H. Shen, Z.B. Xie, A.M. Yue, et al., Expression level of microRNA-195 in the serum of patients with gastric cancer and its relationship with the clinicopathological staging of the cancer. Eur Rev Med Pharmacol Sci, 2016, 20(7): 1283-1287.

[79] C.G. Hou, X.Y. Luo, and G. Li, Diagnostic and Prognostic Value of Serum MicroRNA-206 in Patients with Gastric Cancer. Cell Physiol Biochem, 2016, 39(4): 1512-1520.

[80] J. Zhang, Y. Song, C. Zhang, et al., Circulating MiR-16$5 p$ and MiR-19b-3p as Two Novel Potential Biomarkers to Indicate Progression of Gastric Cancer. Theranostics, 2015, 5(7): 733-745.

[81] V.Y. Shin, E.K. Ng, V.W. Chan, et al., A three-miRNA signature as promising non-invasive diagnostic marker for gastric cancer. Mol Cancer, 2015, 14: 202.

[82] J. Song, Z. Bai, W. Han, et al., Identification of suitable reference genes for qPCR analysis of serum microRNA in gastric cancer patients. Dig Dis Sci, 2012, 57(4): 897-904.

[83] S. Kumar, J. Huang, N. Abbassi-Ghadi, et al., Selected ion flow tube mass spectrometry analysis of exhaled breath for volatile organic compound profiling of esophagogastric cancer. Anal Chem, 2013, 85(12): 6121-6128.

[84] H. Amal, M. Leja, K. Funka, et al., Detection of precancerous gastric lesions and gastric cancer through exhaled breath. Gut, 2016. 65(3): 400-407.

[85] Z.Q. Xu, Y.Y. Broza, R. Ionsecu, et al., A nanomaterialbased breath test for distinguishing gastric cancer from benign gastric conditions. Br J Cancer, 2013, 108(4): 941950.

[86] Y. Chen, Y. Zhang, F. Pan, et al., Breath Analysis Based on Surface-Enhanced Raman Scattering Sensors Distinguishes Early and Advanced Gastric Cancer Patients from Healthy Persons. ACS Nano, 2016.

Copyright $\odot 2016$ Hualin Fu. This is an open-access article distributed under the terms of the Creative Commons Attribution License, which permits unrestricted use, distribution, and reproduction in any medium, provided the original author and source are credited. 